\title{
PSICOLOGIA
}

\section{BREVE PERFIL NEUROPSICOLÓGICO DOS USUÁRIOS DE CRACK DO ESTADO DE RONDÔNIA}

\author{
DOI: http://dx.doi.org/10.31072/rcf.v9i1.561 \\ BRIEF NEUROPSYCHOLOGICAL PROFILE OF USERS CRACK OF THE STATE OF \\ RONDÔNIA
}

Caio Rodrigo Lemos Setúbal'; ;ésica Borges Bergamini²; Victor Hugo Coelho Rocha3; Paulo Renato Vitória Calheiros ${ }^{4}$.

RESUMO: O uso de crack em longo prazo pode causar danos neurológicos severos. Uma maneira de observar a existência de danos neurológicos e do baixo desempenho cognitivo é através da avaliação do estado mental. Objetivos: Esta pesquisa avaliou 228 usuários de crack atendidos em clínicas de recuperação existentes em Rondônia, com tempo médio de uso de nove anos, através do MEEM como objeto de triagem para verificação da condição mental e para verificação da capacidade de responder perguntas mais amplas. Métodos: Foram entrevistados 228 indivíduos (84,6\% pertencentes ao sexo masculino, enquanto $15,4 \%$ sexo feminino), com idades entre 13 e 65 anos, cuja média etária era de 30,28 anos $(\mathrm{DP}=8,9)$, e que, na ocasião da coleta dos dados, se encontravam em regime de internação para o tratamento de dependência química em comunidades terapêuticas localizadas no estado de Rondônia. Resultados e Discussão: Os resultados mostraram que $40 \%$ dos sujeitos estão com índices inferiores e somente 35\% possuem desempenho satisfatório. A média global foi abaixo do ponto de corte. A função mais prejudicada foi à habilidade construtiva e evocação. Conclui-se que é necessário um protocolo clínico que contemple a avaliação do estado mental em dependentes químicos para verificação dos possíveis danos cerebrais e para um melhor atendimento que possa ser de fato reabilitador.

Palavras-chave: Mini exame do estado mental. Avaliação do estado mental. Usuários de crack.

\begin{abstract}
The use of crack in the long term can cause severe neurological damage. One way to observe the existence of neurological damage and low cognitive performance is through mental status assessment. Objectives: This study evaluated 228 crack users attended at existing recovery clinics in Rondônia, with an average time of use of nine years, through the MMSE as an object of screening to verify the mental condition and to verify the
\end{abstract}

\footnotetext{
1 Psicólogo. Mestrando em Psicologia. Universidade Federal de Pernambuco. Autor desta pesquisa. E-mail: caiosetubal@gmail.com. ORCID: http://orcid.org/0000-0002-4726-5263;

2 Professora, Mestra e Orientadora desta pesquisa. E-mail: gpensemagro@hotmail.com. ORCID: https://orcid.org/0000-0003-0598-5366;

3 Acadêmico de Psicologia da Faculdade de Educação e Meio Ambiente - FAEMA. Email: studiovictorocha@gmail.com. ORCID: https://orcid.org/0000-0002-4783-8359;

4 Professor Doutor da Universidade Federal de Rondônia. E-mail: paulocalheiros@unir.br ORCID: https://orcid.org/0000-0003-1897-4180.
} 
capacity to answer broader questions. Methods: A total of 228 individuals (84.6\% male, $15.4 \%$ female), aged 13-65 years, with a mean age of 30.28 years $(S D=8.9)$, were interviewed. and who, at the time of data collection, were hospitalized for the treatment of chemical dependence in therapeutic communities located in the state of Rondônia. Results and Discussion: The results showed that $40 \%$ of subjects had lower indexes and only $35 \%$ had satisfactory performance. The overall mean was below the cutoff point. The most impaired function was constructive ability and evocation. It is concluded that a clinical protocol is required that contemplates the evaluation of mental state in chemical dependents to verify the possible cerebral damages and for a better attendance that can be really rehabilitating.

Words-Keys: Mini mental state examination. Mental state assessment. Crack users.

\section{INTRODUÇÃO}

Para uma compreensão da condição mental do sujeito atendido em clínicas de reabilitação o exame do estado mental apresenta-se como uma ferramenta fundamental que deve ser realizado com todo paciente. Para realização desse exame partem-se do princípio que 0 funcionamento psíquico pode ser analisado em alguns aspectos mais ou menos independentes, embora seja possível argumentar-se que a mente humana é uma integridade indivisível. Porém, através desse exame é possível identificar sinais e sintomas que possibilitarão formular hipóteses diagnósticas e direcionar o raciocínio clínico para síndromes específicas ${ }^{(1,2)}$.

Através desta avaliação o clinico de saúde mental poderá direcionar o melhor tratamento para o sujeito mediante as suas condições mentais, como as funções se apresentam naquele momento. No caso dos pacientes aqui estudados, o exame do estado mental é utilizado para observação do comprometimento cognitivo e danos cerebrais, haja vista que o processo de uso de drogas em longo prazo pode gerar sequelas irreversíveis de cunho cognitivo ou, ainda, desenvolver quadros clínicos neuropsiquiátricos ${ }^{(3,4)}$.

A avaliação do estado mental pode ser realizada de forma breve para fins de rastreio, com 0 intuito de observar sintomas neuropsiquiátricos - mais precisamente demências e condição cognitiva em geral. Para essa avaliação de rastreio o instrumento mais utilizado no Brasil é o Mini Exame do Estado Mental MEEM ${ }^{(1,2)}$.

O MEEM é um teste amplamente utilizado em ambiente clínico para rastreamento de quadros de demências. Contendo diferentes tarefas este instrumento permite avaliar a função cognitiva de indivíduos com ou sem escolaridade formal ${ }^{(5)}$. Traduzido para a língua portuguesa por Bertolucci et al (5), 
avalia orientação no tempo e no espaço, memória de registro, atenção e calculo, memória de evocação, linguagem e habilidade construtiva.

O uso abusivo de drogas, principalmente o uso do crack, pode causar sequelas e danos cognitivos severos. Em virtude dessas possíveis ocorrências, esta pesquisa avaliou, sob forma de triagem, 228 sujeitos atendidos em clínicas de recuperação e comunidades terapêuticas localizadas no Estado de Rondônia através do MEEM.

Conclui-se que o MEEM mostrou-se eficiente e preciso na avaliação cognitiva e foi capaz de identificar possíveis quadros de demências. $A$ análise dos dados revelou que $40 \%$ dos sujeitos da pesquisa estão com índices inferior, 25\% apresentam índice mediano e somente 40\% tiveram índice satisfatório de acordo com o grau de escolaridade ${ }^{(6)}$. A função mental com maior prejuízo foi à habilidade construtiva, caracterizando possível lesão occipital.

Com essa triagem é possível direcionar um tratamento mais eficiente para esses sujeitos, bem como avaliar a eficácia dos tratamentos propostos. Devido à baixa compreensão, retenção e índices de possíveis quadros de demências devido a índices tão baixos, observa-se que o tratamento interventivo deve ser de caráter reabilitador bem mais direcionado a estímulo-resposta, em uma perspectiva comportamental. Além de ser interessante que esses sujeitos sejam submetidos á estimulação cognitiva com 0 intuito de melhorar a compreensão e ter, possivelmente, remissão do quadro demencial (6).

\section{MATERIAIS E MÉTODOS}

Em um estudo de levantamento dos usuários de crack no Estado de Rondônia, utilizou-se o MEEM como objeto de triagem para verificação da condição mental e para verificação da capacidade de responder perguntas mais amplas. A pesquisa avaliou 228 sujeitos atendidos em clínicas de recuperação existentes em Rondônia.

Foram entrevistados 228 indivíduos (84,6\% pertencentes ao sexo masculino, enquanto $15,4 \%$ sexo feminino), com idades entre 13 e 65 anos, cuja média etária era de 30,28 anos $(D P=8,9)$, e que, na ocasião da coleta dos dados, se encontravam em regime de internação para o tratamento de dependência química em comunidades terapêuticas localizadas no estado de Rondônia.

Com relação à escolaridade, 50\% não completaram o ensino fundamental, $10,9 \%$ possuem o ensino fundamental completo, $14,1 \%$ têm o ensino médio incompleto, 13,6\% concluíram o ensino 
médio, 5,5\% não concluíram o ensino superior e $1,4 \%$ apresentaram ensino superior completo. De toda a amostra $92,7 \%$ afirmaram que não estão estudando.

Os participantes voluntários descreveram a opção que melhor representam sua situação atual, tendo $46,2 \%$ da amostra descrito como institucionalizado, $18,6 \%$ procurando por trabalho, $9 \%$ não procura por trabalho, $5,1 \%$ estudante, $4,5 \%$ do lar, $4,5 \%$ aposentado, 2,6\% como incapaz e 9,6\% responderam "outros".

A pesquisa foi aprovada através do Parecer $n^{\circ} 367.336$ emitido pelo Comitê de Ética e Pesquisa da Universidade Federal de Rondônia.

\subsection{Materiais}

Para a coleta dos dados foram empregados os seguintes instrumentos:

1) Termo de Consentimento Livre e Esclarecido: em atendimento à resolução CNS 196/96, todos aqueles que concordaram em participar da pesquisa assinaram um termo no qual eram apresentadas informações acerca dos objetivos e procedimentos para que 0 indivíduo pudesse decidir de forma livre e esclarecida acerca da participação ou não na pesquisa.

2) Mini-Exame de Estado Mental: trata-se de um instrumento utilizado para avaliar a presença de déficits cognitivos. Por meio deste instrumento, são avaliadas as orientações temporal e espacial, cálculo, praxia, memória (imediata ou atenção e evocação), linguagem e habilidades construtiva. Seu escore máximo é 30 e o ponto de corte e definido conforme idade e tempo de escolaridade do sujeito (20).

\subsection{Procedimentos}

Depois de identificadas as comunidades terapêuticas localizadas no estado de Rondônia e destinadas ao atendimento de dependentes químicos, um pesquisador treinado realizou o contato com os responsáveis pelas mesmas a fim de verificar a possibilidade de efetuar a coleta dos dados nessas instituições. $\mathrm{Na}$ sequência, auxiliares de pesquisa previamente treinados e familiarizados com os instrumentos se dirigiam até elas e contataram os internos para verificar a disponibilidade destes em participar da pesquisa.

Após serem esclarecidos acerca dos objetivos, procedimentos e outras informações acerca dos métodos, aqueles que concordaram em participar assinaram o termo de consentimento livre e esclarecido e responderam à bateria de instrumentos, incluindo o MEEM. Os dados foram coletados individualmente, na própria instituição, em sessões com 
duração média de 35 minutos, durante o período de setembro de 2011 e julho de 2012. Os centros de recuperação onde os dados foram coletados eram administrados por instituições religiosas e as internações ocorriam de forma voluntária e sem custos diretos ao indivíduo ou à família.

As informações coletadas foram organizadas em uma planilha eletrônica no programa Statistical Package for the Social Sciences (SPSS), versão 17.0 e o tratamento a elas empregadas constou de testes estatísticos descritivos e de frequências para análise exploratória dos dados e correlação de Pearson aplicado para medidas intervalares ou proporcionais. O nível de significância adotado foi de $p<0,05$ (22).

\section{RESULTADOS E DISCUSSÃO}

O uso de crack no Brasil já é considerado um agravante de ordem de saúde pública, e entre os países emergentes, o Brasil é o maior mercado na América do Sul em números absolutos, com mais de 900.000 usuários. "O crack nada mais é do que a cocaína fumada na forma base livre, facilmente obtida pelo simples processo de aquecimento do cloridrato de cocaína, água e um agente de caráter básico, em geral o bicarbonato de sódio" (8). A cocaína/crack, de acordo com a literatura, é considerada uma das drogas mais utilizadas, assim como o álcool, maconha, merla, óxi e nicotina $(7,8,9)$

Por se tratar de um psicotrópico de fácil acesso e baixo custo, sua difusão tem sido de larga escala, além do fato de seu efeito no SNC ser de intenso prazer, condicionando dessa forma um risco maior para a dependência. Marques et al (10) postulam que:

A dependência a álcool, tabaco e outras drogas - entre elas o crack - é considerada uma síndrome, caracterizada pela presença de um padrão de consumo compulsivo, geralmente voltado para o alivio ou evitação de sintomas de abstinência; esse padrão se mostra mais importante do que parte ou a totalidade das atividades e compromissos sociais realizados pelo individuo, que passa a tratá-los com negligencia ou abandono, a fim de privilegiar o uso. Tal padrão geralmente resulta em tolerância e síndrome de abstinência.

O que torna a característica central da dependência química é o agrupamento de sintomas relacionados à má adaptação do uso de substância (10). Mesmo pouco tempo de uso apresenta grandes chances para dependência e déficits cognitivos relacionados a funcionamento executivo, memória verbal e atenção. A alteração na capacidade de tomadas de decisão aparece com o uso intenso do crack e está associada a problemas no funcionamento do lobo frontal. 
Danos no lobo temporal também podem ocorrer, ocasionando déficits de memória e aprendizagem (11).

Os efeitos neurotóxicos e as consequências comportamentais do uso de substâncias vão depender do tipo de droga ingerida, bem como a forma que a substância é administrada pelo usuário. Os danos provocados por cada uma delas é relativo, respectivo ao tempo de uso e ao período de abstinência. Tendo em vista que cada droga indutora de dependência química é responsável por alterações diferentes ${ }^{(12)}$.

O que motiva um indivíduo ao consumo de drogas são os efeitos que as substâncias psicoativas são capazes de produzir, como por exemplo, provocar a euforia ou aliviar a dor. Em geral as substâncias capazes de provocar tais implicações atuam de maneira diferenciada no circuito do prazer ou de recompensa. A cocaína/crack por sua vez, tem efeitos euforizantes extremamente potentes, podendo levar a dependência após o uso por períodos muito curtos ${ }^{(13)}$. É válido salientar que é necessário que haja vulnerabilidade e suscetibilidade à dependência, seja por condições biológicas, psicológicas, sociais e ambientais (14)

O uso de substâncias psicotrópicas tende a provocar alterações cognitivas e consequentemente

comportamentais ocasionando alterações neuroestruturais, como diminuição no volume, redução na porcentagem de substância cinzenta, alargamento do espaço pericortical e dos ventrículos laterais, morte e diminuição do tamanho dos neurônios e necrose ou atrofia cerebral. Além dos efeitos deletérios produzidos no metabolismo $(11,15,16)$.

É importante salientar que atualmente é proposto que o estado de dependência envolve alterações nos circuitos orbifrontais relacionados a comportamentos repetitivos compulsivos (17).

O uso inicial ou abusivo do crack nunca é isolado, geralmente o seu uso se da em decorrência à utilização a priori de outras substâncias, em média se inicia pelas drogas legais, e com o tempo, mesmo que o indivíduo seja usuário exclusivo do crack, o mesmo parte em busca de outras drogas para que sejam utilizadas no intuito de potencializar os efeitos das substâncias inicialmente utilizadas, minimizar a abstinência ou atenuar indesejáveis do consumo.

Mesmo passando pelo consumo de drogas mais amenas, o usuário de crack mantém o perfil de poliusuário, em que o álcool, o cigarro e a maconha estão 
presentes no quadro de poliuso, acarretando em danos cognitivos severos.

Para a avaliação dos resultados desta pesquisa utilizou-se parâmetros medianos de algumas condições:

- O tempo médio de uso foi de nove anos. Todos os sujeitos relataram usar outras substâncias além do crack em todo esse tempo.

- A escolaridade média foi de ensino fundamental completo.

- A idade média foi 30 anos.
Portanto, de acordo com Folstein (20) o ponto de corte para sujeitos com 30 anos e grau de escolaridade do ensino fundamental completo, para o MEEM deve ser de 26,5 pontos.

O Gráfico 1 mostra que o resultado médio dos sujeitos da pesquisa encontrase abaixo da média. Sob uma visão geral, $40 \%$ dos sujeitos da pesquisa estão com índices inferior, 25\% apresentam índice mediano e somente $40 \%$ tiveram índice satisfatório de acordo com o grau de escolaridade ${ }^{(6)}$.

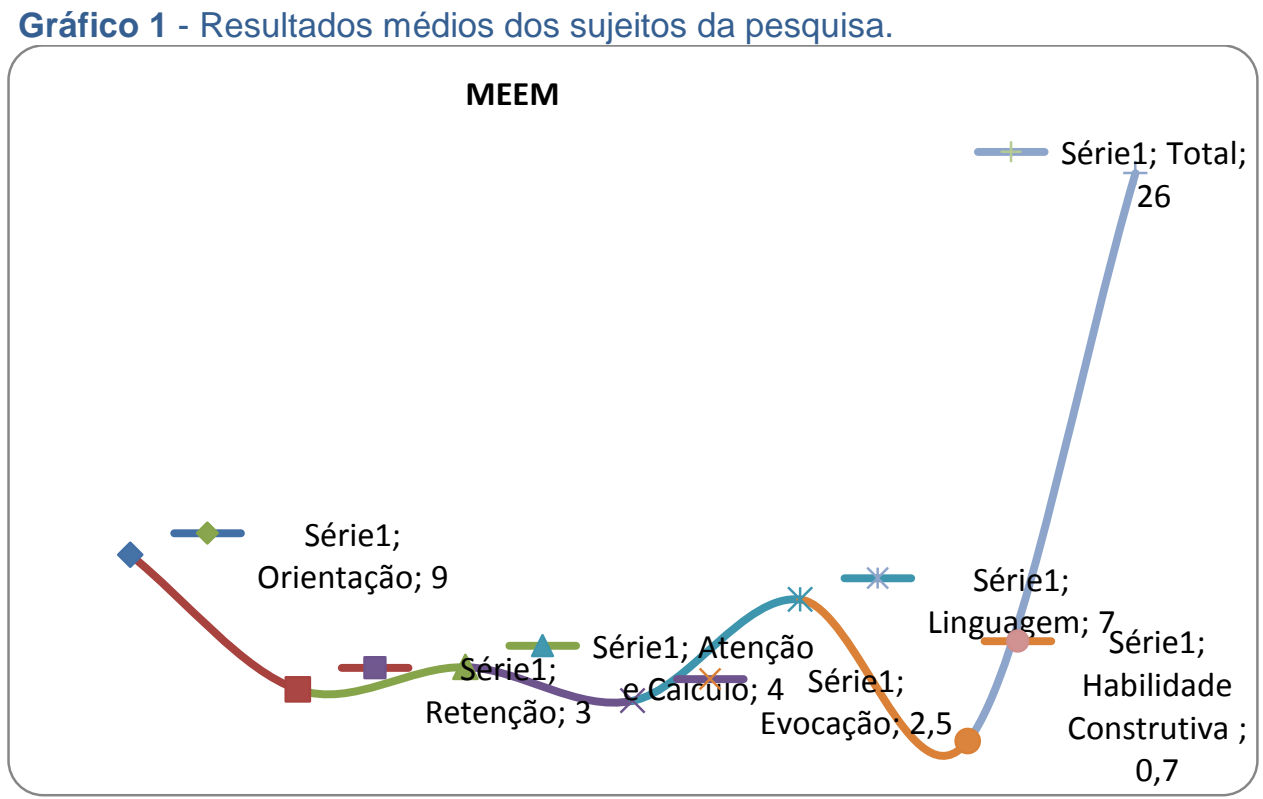

A área mais comprometida foi á habilidade construtiva, uma tarefa relativamente simples, na qual o sujeito precisa reproduzir um desenho de baixo grau de dificuldade que envolve planejamento e coordenação motora.

Observa-se comprometimento menor nas funções de orientação, atenção e calculo evocação e linguagem. $\mathrm{Na}$ atividade de retenção - atividade na qual o sujeito escuta três palavras e repete-as seguidamente, mostram desempenho satisfatório.

Realizando uma análise neuropsicológica, observa-se uma baixa no funcionamento do occipital - através da 
habilidade construtiva, comprometimento na área frontal - dificuldade no planejamento, na concentração e raciocínio, porém a memória operacional apresenta-se preservada, haja vista 0 desempenho na atividade de retenção $(21,3$, 22, 4).

Estes dados confirmam os resultados de pesquisas anteriores no qual os dependentes de cocaína/crack possuem déficits específicos de funções executivas, por exemplo, tomada de decisão e julgamento, e esse comportamento está associado com disfunções de regiões cerebrais pré-frontais específicas. Apresentam desempenho prejudicado em testes de funcionamento do sistema motor e têm tempos de reação mais lentos do que indivíduos não dependentes, ou seja, identificam-se indicadores de comprometimento severo nos usuários desta pesquisa $(19,18)$.

Estudos revelaram que déficits cognitivos associados ao uso crônico de cocaína/crack vêm sendo notados e refletem mudanças nos mecanismos corticais, subcorticais e neuromoduladores subjacentes que embasam a cognição. Indivíduos que são dependentes de cocaína/crack possuem déficits específicos de funções executivas, por exemplo, tomada de decisão e julgamento, e esse comportamento está associado com disfunções de regiões cerebrais préfrontais específicas ${ }^{(19)}$.

Pesquisas realizadas com tomografia por emissão de pósitron e neuroimagem sugerem que a estimulação do sistema dopaminérgico, secundária ao uso crônico de cocaína/crack, ative um circuito que envolve o córtex orbifrontal, o giro do cíngulo, o tálamo e o striatum. Esse circuito está anormal em pessoas com dependência de cocaína/crack e se formula a hipótese de que essa anormalidade contribui para 0 desejo intenso de usar a droga, resultando na perda de controle sobre o direcionamento para usar mais a droga (19).

Investigações clínicas e pré-clínicas fornecem evidências convincentes de prejuízos neurológicos e psiquiátricos persistentes e possível degeneração neuronal associado ao uso crônico do crack. Pessoas com dependência de cocaína/crack exibem desempenho prejudicado em testes do funcionamento do sistema motor e têm tempos de reação mais lentos do que indivíduos não dependentes, ou seja, identificam-se indicadores de comprometimento severo no SNC dos usuários crônicos ${ }^{(18)}$.

Alves, Ribeiro e Castro ${ }^{(19)}$ acentuam que o uso prolongado leva à diminuição da dopamina na sinapse, em decorrência do aumento da metabolização desta e do 
envio de estímulos de liberação menos intensos pelo organismo. Com o uso crônico, há redução do limiar de convulsibilidade a expensas da sensibilização da cocaína, atribuída ao seu componente anestésico.

\section{CONSIDERAÇÕES}

Os usuários de crack do estado de Rondônia apresentam considerável déficit cognitivo. É necessário que as clínicas e comunidades terapêuticas e locais de atendimento a essa população realizem avaliações mais profundas com intuito de verificar com maior precisão o estado mental desses sujeitos.

Analisando os dados do MEEM, observou-se comprometimento significativo na habilidade construtiva, evocação, linguagem, atenção e cálculo. Funcionamento satisfatório nas funções de retenção e orientação temporal e espacial. De uma forma geral os resultados mostram que a média populacional apresentou índices abaixo da linha de corte.

Para uma análise interventiva é importante relatar a necessidade de que os tratamentos realizados nessas instituições estejam organizados através de um caráter reabilitador não somente para longe da condição do uso das drogas, mas para uma melhora das funções mentais lesionados pelo uso prolongado.
Compreender a condição mental do sujeito e os possíveis danos neuropsicológicos causados pelo uso abusivo de drogas traz novas abordagens dinâmicas para 0 tratamento desses pacientes, e para o próprio fechamento de possíveis diagnósticos psiquiátricos.

Pode-se desenvolver

uma abordagem focal, na mudança de aprendizagem, pois se sabe hoje que 0 processo de extinção de uma resposta condicionada não está relacionado a uma destruição de aprendizagem original, mas sim a um novo processo de aprendizagem, no qual novas estruturas neurais adquirem a capacidade de inibir aquelas relacionadas com a resposta condicionada (23).

Desta forma, os sujeitos com tal comprometimento cognitivo precisam de medida interventiva reabilitadora de cunho comportamental e atividades práticas que favoreçam novas construções neuronais com o intuito de promover uma melhora no funcionamento cerebral.

Esta pesquisa traz informações importantes e pode ser observada como uma possível resposta aos questionamentos dos fracassos no atendimento reabilitador dos usuários de drogas. $O$ fracasso existe porque se trabalha com parâmetros errôneos de condições cognitivas. É necessário que as 
clínicas tenham protocolo de tratamento formulado de acordo com a condição cognitiva do sujeito atendido e não um padrão global. É necessário que o sujeito que adentra ao tratamento reabilitador passe por exames mentais, avaliação do estado mental, para verificação real de suas potencialidades e déficits.

E finalizamos esta pesquisa reafirmando a necessidade de que 0 clínico de saúde mental, das mais variadas áreas (terapia ocupacional, fonoaudiologia, fisioterapia, psicologia, nutricionista e médicos) possam pensar no mine exame do estado mental como uma boa ferramenta para o processo de triagem e diagnóstico inicial para direcionamento do tratamento necessário. Compreender a dinâmica neurológica do sujeito permitirá que o profissional tenha uma visão ampla das patologias - seja de lesionados ou não, para que $o$ tratamento tenha um enfoque terapêutico interdisciplinar, que promova de fato a intervenção que o sujeito atendido precisa, e que tenha a melhora desejada.

\section{REFERÊNCIAS}

1. Oliveira JM, Lima RP. O Exame do Estado Mental. Pelotas: Universitária; 2000.

2. Cordioli AV, Zimmermann HH, Kessler F. Rotina de Avaliação do Estado Mental. 2004. [citado em 01 de setembro de 2017]. Disponível em: http://www.ufrgs.br/psiquiatria/psiq/Avalia\% C3\%A7\%C3\%A30\%20\%20do\%20Estado \%20Mental.pdf.

3. Pawlowski J. Instrumento de Avaliação Neuropsicologico Breve NEUPSILIN: Evidências de Validade de Construto e de Validade Incremental à Avaliação Neuropsicologia. [Tese de Doutorado]. Rio Grande do Sul: Universidade Federal do Rio Grande do Sul - UFRGS; 2011. [citado em 20 de agosto de 2017]. Disponível em: http://www.lume.ufrgs.br/bitstream/handle/ 10183/27835/000765892. pdf? sequence $=1$.

4. Caixeta L, Ferreira SB. Manual de Neuropsicologia. $1^{\underline{a}}$ ed. São Paulo: Atheneu; 2012.
5. Bertolucci PHF, Bruck S, Campacci SR, Juliano Y. O Mini-Exame do Estado Mental em uma População Geral: Impacto da Escolaridade. Rev Arquivos de NeuroPsiquiatria 1994; 52(1): 1-7.

6. Brucki SMD, Nitrini $R$, Caramelli $P$, Bertolucci PHF, Okamoto IH. Sugestões para o uso do Mini-Exame do Estado Mental no Brasil. Rev Arquivos de NeuroPsiquiatria 2003, 61(3):777-781. [citado em 01 de setembro de 2017]. Disponível em: http://www.scielo.br/pdf/\%0D/anp/v61n3b/1 7294.pdf.

7. Kaplan BJ, Sadock VA. Compêndio de Psiquiatria. Ciência do Comportamento e Psiquiatria Clínica. 9a ed. Porto Alegre (RS): Artmed; 2007.

8. Washton AM, Zweben JE. Prática Psicoterápica Eficaz dos Problemas com Álcool e Drogas. Tradução: Armando $M$. Porto Alegre (RS): Artmed; 2009. [citado em 20 de agosto de 2017]. Disponível em: https://books.google.com.br/books?hl=pt$B R \& \mid r=\& i d=0 x-$

8RidqpVQC\&oi=fnd\&pg=PP5\&dq=61.\%09 Washton+AM,+Zweben+JE.+Pr\%C3\%A1ti ca+Psicoter\%C3\%A1 pica+Eficaz+dos+Pro 
blemas+com+\%C3\%81lcool+e+Drogas. $+\mathrm{T}$ radu\%C3\%A7\%C3\%A3o:+M.+Armando.+ Porto+Alegre+(RS):+Artmed, $+2009 . \&$ ots $=h$ AsO4IM0nX\&sig=b9Jca3-

4ZwODbAWb7Q3MMqJJtuA\#v=onepage\& $\mathrm{q} \& \mathrm{f}=\mathrm{false}$.

9. Stahl SM. Psicofarmacologia: Bases neurocientíficas e aplicações práticas. 3aㅗ ed. Rio de Janeiro: Guanabara Koogan; 2010.

10. Marques ACPR, Ribeiro M, Laranjeira RR, Andrada NC. Abuso e Dependência: Crack. Rev da Associação Médica Brasileira 2012; 58(2): 141-153. [citado em 01 de setembro de 2017]. Disponível em: http://www.scielo.br/pdf/ramb/v58n2/v58n2 a08.pdf.

11. Almeida PP, Monteiro MF. Neuropsicologia e Dependência Química. In: Diehl A, Cordeiro DC, Laranjeira R, colaboradores. Dependência Química: Prevenção, Tratamento e Políticas Públicas. Porto Alegre (RS): Artmed; 2011. p. 98-105.

12. Graeff FG. Abuso e Dependência de Drogas. In: Graeff FG, Guimarães FS, organizadores. Fundamentos de Psicofarmacologia. $2^{\underline{a}}$ ed. São Paulo (SP): Atheneu; 1999.

13. Fonseca VAS, Lemos T. Farmacologia na Dependência Química. In: Diehl A, Cordeiro DC, Laranjeira R, colaboradores. Dependência Química: Prevenção, Tratamento e Políticas Públicas. Porto Alegre (RS): Artmed; 2011. p. 25-34.

14. Andrade AG, Anthony JC. Álcool e suas Consequências: Uma Abordagem Multiconceitual. Barueri: Minha Editora; 2009.

15. Goldstein RZ, Volkon ND. Drug addiction ans its underlying neurobiological basis: neuroimaging evidence for the involvement of the frontal cortex. Journal of Psychiatric Research 2002; 156(10): 164252.
16. Bartozkis $G$, Beckson $M$, Lu PH, Edwards N, Rapoport R, Wiseman E, et al. Age-related brain volume reductions in amphetamine and cocaine addicts and normal controls: implications for addiction research. Psychiatry Research 2000; 98(2): 93-102.

17. Organização Mundial da Saúde OMS. Neurociência do uso e da dependência de substâncias psicoativas. São Paulo: Roca; 2006. [citado em 01 de setembro de 2017]. Disponível em: http://apps.who.int/iris/bitstream/10665/426 66/2/9788572416665_por.pdf.

18. Tomasi D, Volkon ND, Wang $R$, Carrillo JH, Maloney T. Disrupted functional connectivity with dopaminergic midbrain in cocaine abusers. PloS one 2010; 5(5).

19. Alves HNP, Ribeiro M, Castro DS. Cocaína e Crack. In: Diehl A, Cordeiro DC, Laranjeira R, colaboradores. Dependência Química: Prevenção, Tratamento e Políticas Públicas. Porto Alegre (RS): Artmed; 2011. p. 170-179.

20. Folstein MF, Folstein SE, Mchugh PR. Mini-Mental State: a practical method for grading the cognitive state of patients for clinician. Journal of Psychiatric Research 1975; 12(3): 189-198.

21. Malloy-Diniz LF, Fuentes D, Mattos $P$, Abreu N, colaboradores. Avaliação Neuropsicológica. Porto Alegre (RS): Artmed, 2010. [citado em 01 de setembro de 2017]. Disponível em: https://books.google.com.br/books?hl=pt$B R \& \mid r=\& i d=X g n S A D 3 S m t 4 C \& o i=f n d \& p g=P$ $\mathrm{R} 5 \& \mathrm{dq}=54 . \% 09 \mathrm{MALLOY}$ -

DINIZ,+Leandro+F\%3B+FUENTES,+Danie $1 \% 3 \mathrm{~B}+\mathrm{MATTOS},+\mathrm{Paul0} \% 3 \mathrm{~B}+\mathrm{ABREU},+\mathrm{Ne}$ ander.+Avalia\%C3\%A7\%C3\%A30+Neurop sicol\%C3\%B3gica.+Porto+Alegre+(RS):+A rtmed, +2010 .\&ots=EHNTuf3vGa\&sig=Uhp83LXwmSiJpY8fDMlbGQd7kc\#v=onepage $\& q \& f=f a l s e$.

22. Bear MF, Connors BW, Paradiso MA. Neurociências: Desvendando o Sistema 
Nervoso. $4^{\mathrm{a}}$ ed. Porto Alegre (RS): Artmed; 2017.

23. Cordioli AV, Zimmermann $\mathrm{HH}$, Kessler F. Rotina de Avaliação do Estado Mental. 2004. [citado em 01 de setembro de 2017].
Disponível em: http://www.ufrgs.br/psiquiatria/psiq/Avalia\% C3\%A7\%C3\%A30\%20\%20do\%20Estado $\% 20$ Mental.pdf.

\section{Como citar (Vancouver)}

Setúbal CRL, Bergamini GB, Rocha VHC, Calheiros PRV. Breve perfil neuropsicológico dos usuários de crack do estado de Rondônia. Rev Cient Fac Educ e Meio Ambiente [Internet]. 2018;9(1):289-300. DOI: http://dx.doi.org/10.31072/rcf.v9i1.561 